\title{
New stability criteria for stochastic perturbed singular systems in mean square
}

\author{
Tomás Caraballo · Faten Ezzine · Mohamed Ali Hammami [
}

Received: 18 January 2021 / Accepted: 9 June 2021 / Published online: 24 June 2021

(C) The Author(s), under exclusive licence to Springer Nature B.V. 2021

\begin{abstract}
In this paper, we investigate the problem of stability of time-varying stochastic perturbed singular systems by using Lyapunov techniques under the assumption that the initial conditions are consistent. Sufficient conditions on uniform exponential stability and practical uniform exponential stability in mean square of solutions of stochastic perturbed singular systems are obtained based upon Lyapunov techniques. Furthermore, we study the problem of stability and stabilization of some classes of stochastic singular systems. Finally, we provide numerical examples to validate the effectiveness of the main results of this paper.
\end{abstract}

Keywords Linear time-varying singular systems . Standard canonical form - Consistent initial conditions . Lyapunov function · Itô formula · Brownian motion ·

The research of Tomás Caraballo has been partially supported by the Spanish Ministerio de Ciencia, Innovación y Universidades (MCIU), Agencia Estatal de Investigación (AEI) and Fondo Europeo de Desarrollo Regional (FEDER) under the project PGC2018-096540-B-I00, and by FEDER and Junta de Andalucía (Consejería de Economía y Conocimiento) under projects US-1254251, and P18-FR-4509.

\section{T. Caraballo}

Depto. Ecuaciones Diferenciales y Análisis Numérico, Facultad de Matemáticas, Universidad de Sevilla, c/ Tarfia s/n, Sevilla 41012, Spain

F. Ezzine · M. A. Hammami $(\varangle)$

Faculty of Sciences of Sfax, Department of Mathematics, University of Sfax, Sfax, Tunisia

e-mail: MohamedAli.Hammami@fss.rnu.tn
Nontrivial solution · Practical exponential stability in mean square $\cdot$ Stabilization

Mathematics Subject Classification Primary

37B55 · Secondary 34D20

\section{Introduction}

Differential-algebraic equations (DAEs) are a combination of differential equations along with algebraic constraints. This class of systems is also called singular systems, degenerate systems, generalized systems, descriptor systems, semi-state systems, or generalized state-space systems.

Singular systems representation has been used as a perfect tool to model a wide variety of problems, such as electrical engineering, aircraft dynamics, robotics, economics, optimization problems, chemistry, biology, etc., which usually cannot be formed by standard ordinary differential equations (ODEs).

As it is widely known, environmental noise exists and cannot be ignored. In fact, some parameters of dynamical systems may be existed or perturbed by some environmental noise. Then, we obtain a generalization of differential-algebraic equations (DAEs) and stochastic differential equations (SDEs). This class of systems represents stochastic differential-algebraic equations (SDAEs).

Indeed, it is essential to analyze whether the presence of some random terms in the equations of the 
models may produce a very different behavior of their solutions. Although there exists a wide literature on this topic, we would like to mention [10,16,21,24,25,28].

In this paper, we consider the combination of Itô stochastic representation and linear time-varying singular form to consider a large class of realistic systems that are modeled with stochastic differential-algebraic equations (SDAEs).

Because of the difficulty arising in analysis, few results are concerned with the stability of stochastic perturbed linear time-varying singular systems.

The stability theory of linear differential-algebraic systems is an active research topic. Different authors tackled the question of stability and stabilization of time-invariant singular stochastic systems, see $[6,26$, 28-30]. However, there have been few results on the stability of linear time-varying singular systems in the past few decades, and we here mention $[5,7,8,23]$ among others.

The approach of Lyapunov functions is one of the most efficient ones for the investigation of the stability of dynamical systems, see [1,2], in particular, of stochastic systems, see $[10,11,17,27]$.

Lyapunov stability of stochastic singular systems has attracted also the attention of several authors, and we here mention $[5,6,13]$ among others.

When the origin is not a trivial solution, we investigate the stability of the SDEs with respect to a small neighborhood of the origin. In that sense, we can analyze the ultimate boundedness of the solutions of the perturbed system or the possibility of proving the convergence of the solutions toward a small ball centered at the origin. Several results on the stability of the nontrivial solution of stochastic systems are proposed in $[1,11,12]$.

To the best of our knowledge, no work has been published about the practical stability of linear timevarying stochastic perturbed singular systems. Our main objective in this paper is to extend the results in [12], which investigated the practical stability of linear time-invariant perturbed stochastic systems, to the case of linear time-varying stochastic perturbed systems.

Based upon the method of Lyapunov functions and generalized Gronwall inequalities, we establish some criteria for uniform exponential stability and practical uniform exponential stability in mean square of a class of linear time-varying stochastic perturbed systems.

The organization of the paper is as follows. In Section 2, we recall some necessary preliminaries and results about linear time-varying singular systems which are transferable into a standard canonical form. In Section 3, we discuss the problem of existence and uniqueness of our system. In Section 4, we prove some sufficient conditions ensuring uniform exponential stability and practical uniform exponential stability in mean square of linear time-varying stochastic perturbed singular systems based on the method of Lyapunov under the presumption that the initial conditions are consistent. In Section 5, we state sufficient conditions for uniform and practical uniform exponential stabilization in mean square of stochastic singular systems. In Section 6, we exhibit some illustrative examples to show the applicability of our abstract theory. Finally, some conclusions and prospects are given in the last section.

\section{Preliminary results}

Throughout this paper, unless otherwise specified, we use the following notations:

\section{Notations:}

$\operatorname{ker} D$ : The kernel of the matrix $D \in \mathbb{R}^{m \times n}$.

$\operatorname{im} D$ : The image of the matrix $D \in \mathbb{R}^{m \times n}$.

$\mathbb{G} \mathbb{I}_{n}(\mathbb{R})$ : The general linear group of degree n, i.e., the set of all invertible $n \times n$ matrices over $\mathbb{R}$.

$\mathbb{C}(J, S)$ : The set of continuous functions $g: J \rightarrow$ $S$ from an open set $J \subseteq \mathbb{R}$ to a vector space S.

$\mathbb{C}^{k}(J, S)$ : The set of k-times continuously differentiable functions g: $J \rightarrow S$ from an open set $J \subseteq \mathbb{R}$ to a vector space $S$.

dom $g$ : The domain of the function $g$.

$I_{n}$ : Identity matrix in $\mathbb{R}^{n \times n}$.

$\|x\|:=\sqrt{x^{T} x}:$ Euclidean norm of $x \in \mathbb{R}^{n}$.

$\|D\|:=\sup \{\|D x\| \mid\|x\|=1\}$, induced matrix norm of $\mathrm{D} \in \mathbb{R}^{m \times n}$.

$\|g\|_{\infty}:=\sup \{\|g(t)\| \mid t \in \operatorname{dom} g\}$, the infinity norm of the function $g$.

Now, let us consider the following linear timevarying continuous singular system:

$E(t) \dot{x}(t)=A(t) x(t), \quad x\left(t_{0}\right)=x_{0}$, 
where $x(t) \in \mathbb{R}^{n}$ is the system state vector, $x\left(t_{0}\right)=$ $x_{0} \in \mathbb{R}^{n}$ is the initial condition, with $(E, A) \in$ $\mathbb{C}\left(\mathbb{R}_{+}, \mathbb{R}^{n \times n}\right)^{2}$, where $E$ is a singular matrix.

\subsection{Standard canonical form}

In this paragraph, we introduce the concept of consistent initial values, and the subclass of DAEs $(E, A)$ which are transferable into a standard canonical form. Furthermore, we recall some needed properties for the subsequent sections. For extra details, we refer the reader to see $[19,29]$.

Definition 2.1 A pair $\left(t_{0}, x_{0}\right) \in \mathbb{R}_{+} \times \mathbb{R}^{n}$ is called a pair of consistent initial values of the linear timevarying singular system (2.1) if there exists a solution $x(\cdot)$ of (2.1), with $t_{0} \in \operatorname{dom} x(\cdot)$ and $x\left(t_{0}\right)=x_{0}$.

In the sequel, we denote by $\mathcal{W}$ the set of all pairs of consistent initial values of the linear time-varying singular system (2.1). In addition, for $t_{0} \in \mathbb{R}_{+}$,

$\mathcal{W}\left(t_{0}\right)=\left\{x_{0} \in \mathbb{R}^{n}:\left(t_{0}, x_{0}\right) \in \mathcal{W}\right\}$

$\mathcal{W}\left(t_{0}\right)$ is the linear subspace of initial values, which are consistent at time $t_{0}$.

Notice that, if $x: J \rightarrow \mathbb{R}^{n}$ is a solution of the system (2.1), then $x(t) \in \mathcal{W}(t)$ for all $t \in J$.

Definition 2.2 The DAEs $\left(E_{1}, A_{1}\right)$ and $\left(E_{2}, A_{2}\right) \in$ $\mathbb{C}\left(\mathbb{R}_{+}, \mathbb{R}^{n \times n}\right)^{2}$ are said to be equivalent, if there exist $S \in \mathbb{C}\left(\mathbb{R}_{+}, \mathbb{G}_{n}(\mathbb{R})\right)$ and $T \in \mathbb{C}^{1}\left(\mathbb{R}_{+}, \mathbb{G I}_{n}(\mathbb{R})\right)$, such that

$E_{2}=S E_{1} T, \quad A_{2}=S A_{1} T-S E_{1} \frac{d}{d t}(T)$,

and we write $\left(E_{1}, A_{1}\right) \sim\left(E_{2}, A_{2}\right)$.

Definition 2.3 The system (2.1) is said to be transferable into a standard canonical form (SCF), if there exist $S \in \mathbb{C}\left(\mathbb{R}_{+}, \mathbb{G I}_{n}(\mathbb{R})\right), T \in \mathbb{C}^{1}\left(\mathbb{R}_{+}, \mathbb{G I}_{n}(\mathbb{R})\right), b>0$, such that

$(E, A) \sim\left[\left(\begin{array}{cc}I_{b} & 0 \\ 0 & N\end{array}\right),\left(\begin{array}{cc}J & 0 \\ 0 & I_{n-b}\end{array}\right)\right]$,

where $J: \mathbb{R}_{+} \rightarrow \mathbb{R}^{b \times b}$ and $N: \mathbb{R}_{+} \rightarrow \mathbb{R}^{(n-b) \times(n-b)}$, which is pointwise strictly lower triangular matrix. A matrix $N$ is called pointwise strictly lower triangular, if all entries of $N(t)$ on the diagonal and above are zero for all $t \in \mathbb{R}_{+}$.
Now, we are in a position to characterize the set of consistent initial conditions DAEs $(E, A)$, for the class which are transferable into a standard canonical form.

Proposition 2.1 [29] Suppose that the DAE $(E, A) \in$ $\mathbb{C}\left(\mathbb{R}_{+}, \mathbb{R}^{n \times n}\right)^{2}$ is transferable into a standard canonical form. Then,

$\left(t_{0}, x_{0}\right) \in \mathcal{W} \Longleftrightarrow x_{0} \in \operatorname{imT}\left(t_{0}\right)\left(\begin{array}{c}I_{b} \\ 0\end{array}\right)$.

\section{Existence and uniqueness problem}

Assume that some parameters of the deterministic linear time-varying singular system (2.1) are excited or perturbed by some environmental noise (see Oksendal [18], Mao [20] for more details).

Then, the stochastic perturbed singular system is described by the following Itô equation:

$E(t) d x(t)=A(t) x(t) d t+E(t) \Pi(t) f(t, x(t)) d B_{t}$,

where $x\left(t_{0}\right)=x_{0} \in \mathbb{R}^{n}$ is the initial condition of the system, $B_{t} \in \mathbb{R}$ is a standard Brownian motion defined on a complete probability space $(\Omega, F, P)$ with $B_{0}=$ 0 . $(E, A) \in \mathbb{C}\left(\mathbb{R}_{+}, \mathbb{R}^{n \times n}\right)^{2}$, where $\mathrm{E}$ is a singular matrix. $\Pi \in \mathbb{C}\left(\mathbb{R}_{+}, \mathbb{R}^{n \times n}\right)$, such that $\operatorname{im} \Pi(t)=\mathcal{W}(t)$ for all $t \in \mathbb{R}_{+}$, and $f: \mathbb{R}_{+} \times \mathbb{R}^{n} \rightarrow \mathbb{R}^{n}$.

Remark 3.1 $E \Pi f$ is a structured perturbation and secures that the stochastic perturbation does not affect by the algebraic part in the system. Different authors tackled the approach of structured perturbation for linear algebraic differential equations, see [4, 12]. Indeed, $E \Pi f$ is a structured perturbation that guarantees consistency with the SDAEs (3.1), that is

$E(t) \Pi(t) f(t, x(t)) \in \mathcal{W}(t)$, for all $t \geq 0$.

Our target now is to prove the existence and uniqueness of solution to our problem.

For the well-posedness of system (3.1), we impose the following assumptions:

1. $(E, A) \in \mathbb{C}\left(\mathbb{R}_{+}, \mathbb{R}^{n \times n}\right)^{2}$ is transferable into a standard canonical form.

2. The function $f(t, x)$ satisfies the following relations $\forall t \geq 0, \forall x, \tilde{x} \in \mathbb{R}^{n}$,

$$
\begin{aligned}
\|f(t, x)\| & \leq K_{1}\|x\|+K_{2}, \\
\|f(t, x)-f(t, \tilde{x})\| & \leq K_{3}\|x-\tilde{x}\|,
\end{aligned}
$$


where $K_{1}, K_{2}$ and $K_{3}$ are given strictly positive constants.

Now, we state and prove the following result for the existence and uniqueness of the solution to the stochastic perturbed singular system (3.1).

Theorem 3.2 Under assumptions (1) and (2), the time-varying stochastic perturbed singular system (3.1) has a unique global solution.

In order to prove Theorem 3.2, we need to recall the following crucial lemma, which deals with the subsystem $N(t) d x(t)=x(t) d t$, which is called a pure differential-algebraic equation.

Lemma 3.3 [29] Let $N(\cdot) \in \mathbb{C}\left(\mathbb{R}_{+}, \mathbb{R}^{(n-b) \times(n-b)}\right)$ be pointwise strictlylower triangular matrix. Then, $x(\cdot)=$ 0 is the unique global solution of the pure differentialalgebraic equation

$N(t) d x(t)=x(t) d t$.

Proof of Theorem 3.2. $(E, A) \in \mathbb{C}\left(\mathbb{R}_{+}, \mathbb{R}^{n \times n}\right)^{2}$ is transferable into a standard canonical form, and then, there exist $S \in \mathbb{C}\left(\mathbb{R}_{+}, \mathbb{G I}_{n}(\mathbb{R})\right), T \in \mathbb{C}^{1}\left(\mathbb{R}_{+}, \mathbb{G I}_{n}(\mathbb{R})\right)$, such that:

$$
\begin{aligned}
& S(t) E(t) T(t)=\left(\begin{array}{cc}
I_{b} & 0 \\
0 & N(t)
\end{array}\right), \\
& S(t) A(t) T(t)-S(t) E(t) \frac{d}{d t}(T(t))=\left(\begin{array}{cc}
J(t) & 0 \\
0 & I_{n-b}
\end{array}\right),
\end{aligned}
$$

where $J: \mathbb{R}_{+} \rightarrow \mathbb{R}^{b \times b}$ and $N: \mathbb{R}_{+} \rightarrow \mathbb{R}^{(n-b) \times(n-b)}$, which is pointwise strictly lower triangular matrix. Thus, we obtain

$$
\begin{aligned}
& S(t) E(t) d x(t) \\
& \quad=S(t) A(t) x(t) d t+S(t) E(t) \Pi(t) f(t, x(t)) d B_{t}, \\
& S(t) E(t) T(t) T^{-1}(t) d x(t) \\
& \quad=S(t) A(t) T(t) T^{-1}(t) x(t) d t \\
& \quad+S(t) E(t) \Pi(t) f(t, x(t)) d B_{t} .
\end{aligned}
$$

Let $z(t)=T^{-1}(t) x(t)=\left(z_{1}^{T}(t), \quad z_{2}^{T}(t)\right)^{T}$, where $z_{1}(t) \in \mathbb{R}^{b}$ and $z_{2}(t) \in \mathbb{R}^{n-b}$.
Then, it follows that

$$
\begin{gathered}
S(t) E(t) T(t)\left(d z(t)-\frac{d}{d t}\left(T(t)^{-1}\right) x(t) d t\right) \\
=S(t) A(t) T(t) z(t) d t \\
\quad+S(t) E(t) \Pi(t) f(t, T(t) z(t)) d B_{t} .
\end{gathered}
$$

That is,

$$
\begin{aligned}
& S(t) E(t) T(t) d z(t)-S(t) E(t) T(t) \frac{d}{d t}\left(T(t)^{-1}\right) x(t) d t \\
& =S(t) A(t) T(t) z(t) d t \\
& \quad+S(t) E(t) \Pi(t) f(t, T(t) z(t)) d B_{t} .
\end{aligned}
$$

In view of $\frac{d}{d t}\left(T(t)^{-1}\right)=-T(t)^{-1} \frac{d}{d t}(T(t)) T(t)^{-1}$, which follows from differentiation of $I=T^{-1}(t) T(t)$. Consequently, we obtain

$$
\begin{aligned}
S(t) & E(t) T(t) d z(t) \\
& +S(t) E(t) T(t) T^{-1}(t) \frac{d}{d t}(T(t)) T(t)^{-1} x(t) d t \\
= & S(t) A(t) T(t) z(t) d t \\
& +S(t) E(t) \Pi(t) f(t, T(t) z(t)) d B_{t} .
\end{aligned}
$$

Thus, we have

$$
\begin{aligned}
& S(t) E(t) T(t) d z(t) \\
& \quad+S(t) E(t) \frac{d}{d t}(T(t)) z(t) d t=S(t) A(t) T(t) z(t) d t \\
& \quad+S(t) E(t) \Pi(t) f(t, T(t) z(t)) d B_{t} .
\end{aligned}
$$

Hence, we see that

$$
\begin{aligned}
& S(t) E(t) T(t) d z(t) \\
& =\left(S(t) A(t) T(t)-S(t) E(t) \frac{d}{d t}(T(t))\right) z(t) d t \\
& \quad+S(t) E(t) \Pi(t) f(t, T(t) z(t)) d B_{t} .
\end{aligned}
$$

Based upon the form of SET (3.5), we may always choose $\Pi$ in the following form:

$$
\Pi=\left(\begin{array}{cc}
I_{b} & 0 \\
0 & 0_{n-b}
\end{array}\right) .
$$


Consequently, for $f=\left(\begin{array}{l}f_{1} \\ f_{2}\end{array}\right)$, we obtain $\Pi f=$ $\left(\begin{array}{c}f_{1} \\ 0\end{array}\right)$, that is the perturbation term affects only the differential part of the SDAE (3.1).

Taking into account (3.6) and (3.5), we obtain

$$
\begin{aligned}
& \left(\begin{array}{cc}
I_{b} & 0 \\
0 & N(t)
\end{array}\right)\left(\begin{array}{l}
d z_{1}(t) \\
d z_{2}(t)
\end{array}\right) \\
& =\left(\begin{array}{cc}
J(t) & 0 \\
0 & I_{n-b}
\end{array}\right)\left(\begin{array}{l}
z_{1}(t) \\
z_{2}(t)
\end{array}\right) d t \\
& \quad+S(t) E(t)\left(\begin{array}{c}
f_{1}(t, T(t) z(t)) \\
0
\end{array}\right) d B_{t} .
\end{aligned}
$$

Let $\hat{f}_{1}(t, z(t))=S(t) E(t)\left(\begin{array}{c}f_{1}(t, T(t) z(t)) \\ 0\end{array}\right)$, which in turn gives

$$
\begin{aligned}
d z_{1}(t) & =J(t) z_{1}(t) d t+\hat{f}_{1}(t, z(t)) d B_{t}, \\
N(t) d z_{2}(t) & =z_{2}(t) d t .
\end{aligned}
$$

Since the function $f(t, x)$ satisfies conditions (3.2) and (3.3), then we obtain the conditions of the usual existence and uniqueness theorem for SDEs (see, e.g., $[18,20]$, for the regular $\operatorname{SDE}(3.7))$ :

- Lipschitz condition: The coefficient $\hat{f}_{1}(t, z):=$ $S(t) E(t)\left(\begin{array}{c}f_{1}(t, T(t) z) \\ 0\end{array}\right)$ is

Lipschitz-continuous with respect to $z_{1}$ with a constant $L_{\hat{f}_{1}} \leq K_{3}\|S\|_{\infty}\|E\|_{\infty}\|T\|_{\infty}$, where $K_{3}$ is the Lipschitz constant for the function $f$.

- Growth condition: Since $f$ depends continuously on $t$, also $\hat{f}_{1}$ depends continuously on $t$. Hence, the growth condition follows from the global Lipschitz condition.

Applying the existence and uniqueness theorem for SDEs to the regular stochastic equation (3.7), then the regular SDE (3.7) possesses a unique global solution on the interval $[0,+\infty)$. Moreover, in view of Lemma 3.3 , it follows that the pure differential algebraic equation (3.8) has a unique global solution $z_{2}(\cdot)=0$. Thus, $z(t)=\left[z_{1}(\cdot)^{T}, 0\right]^{T}$, with $z\left(t_{0}\right)=\left(\begin{array}{c}z_{1}\left(t_{0}\right) \\ 0\end{array}\right)$. Eventually, we obtain $x(t)=T(t) z(t)$ is the unique global solution to (3.1) on $t \geq 0$.

Remark 3.4 The assumptions (3.2) and (3.3) for $f(t, x)$ (linear growth condition and global Lipschitz condition) are used in the proof of Theorem 3.2 to ensure that the function $\widehat{f_{1}}(t, x)$ satisfies the same assumptions. In this way, we can always confirm that the regular SDE (3.7) possesses a unique global solution. Thus, the SDAE (3.1) possesses a unique global solution. The above conditions can be weakened by imposing a Lyapunov-like condition and a Local Lipschitz condition. But in this case, we cannot guarantee, in general, that the solution of system (3.1) is globally defined.

Remark 3.5 In this paper, we restrict ourselves to study the problem of stability of a stochastic perturbed singular system, where its associated unperturbed nominal system (2.1) is transferable into a standard canonical form.

Systems transferable into SCF have been introduced by Campbell [9] almost 30 years. Since then, many other approaches to time-varying linear DAEs proposed, see [19,29].

This class is a time-varying generalization of timeinvariant singular system, where the corresponding matrix pencil is regular. The following proposition shows that transferability into SCF for linear timevarying DAEs $(E, A)$ is a generalization of regularity for linear time-invariant DAEs, where $E, A \in \mathbb{R}^{n \times n}$, i.e., $\operatorname{det}(s E-A) \neq 0, \quad s \in \mathbb{C}$.

Proposition 3.6 [29] For $E, A \in \mathbb{R}^{n \times n}$, we have $(E, A)$ is transferable into $S C F \Longleftrightarrow(E, A)$ is regular.

Remark 3.7 Here, we should mention that in [12] we study the stability of the stochastic perturbed singular system for which its associated unperturbed system is a linear time-invariant singular system, where the corresponding matrix pencil is regular. Our main objective in this paper is to extend the results in [12] to the timevarying case where it is transferable into a standard canonical form.

\section{Stability analysis}

Wealthy historic background recorded in the investigation of the stability of linear time-invariant singular systems, where $E \in \mathbb{R}^{n \times n}$ and $A \in \mathbb{R}^{n \times n}$ within the method of Lyapunov, see [9,13-15].

For linear time-invariant singular systems $(E, A) \in$ $\left(\mathbb{R}^{n \times n}\right)^{2}$ it is well known that one seeks for solutions $P, Q \in \mathbb{R}^{n \times n}$ of the Lyapunov equation

$A^{T} P E+E^{T} P A=-Q$, 
and the corresponding Lyapunov function candidate is the following

$V: \mathcal{W} \backslash\{0\} \rightarrow \mathbb{R}, \quad x \longmapsto(E x)^{T} P(E x)$,

where $\mathcal{W} \backslash\{0\}=\mathcal{W}(t)$, for all $t \in \mathbb{R}_{+}$.

For linear time-varying singular systems $(E, A) \in$ $\mathbb{C}\left(\mathbb{R}_{+}, \mathbb{R}^{n \times n}\right)^{2}$, the analogous Lyapunov function is the following:

$V: \mathcal{W} \rightarrow \mathbb{R}, \quad(t, x) \mapsto(E(t) x)^{T} P(t)(E(t) x)$.

T. Berger and A. IIchman [5] generalized the Lyapunov equation (4.1) for linear time-varying singular systems (2.1), which are transferable into a standard canonical form (SCF), such that for all $Q(\cdot) \in$ $\mathbb{C}\left(\mathbb{R}_{+}, \mathbb{R}^{n \times n}\right)$, there exists $P(\cdot) \in \mathbb{C}\left(\mathbb{R}_{+}, \mathbb{R}^{n \times n}\right)$ solves the following equation:

$$
\begin{aligned}
x^{T} & {\left[A^{T}(t) P(t) E(t)+E^{T}(t) P(t) A(t)\right.} \\
& \left.+\frac{d}{d t}\left(E^{T}(t) P(t) E(t)\right)\right] x=-Q(t), \quad \forall(t, x) \in \mathcal{W} .
\end{aligned}
$$

\subsection{Exponential stability of the stochastic perturbed singular system in mean square}

In this section, we assume that the perturbation $f$ vanishes at zero, that is, $f(t, 0)=0, \forall t \geq 0$, which allows us to analyze the stability properties of the null solution to system (3.1) based upon the Lyapunov direct method.

We state the definition of exponential stability in mean square of stochastic perturbed singular system (3.1).

Definition 4.1 The stochastic perturbed singular system (3.1) is said to be uniformly exponentially stable in mean square, if there exist positive constants $\alpha_{1}$ and $\alpha_{2}$, such that for all $\left(t_{0}, x_{0}\right) \in \mathcal{W}$,

$\mathbb{E}\left(\|x(t)\|^{2}\right) \leq \alpha_{1}\left\|x_{0}\right\|^{2} e^{-\alpha_{2}\left(t-t_{0}\right)}, \quad$ for all $t \geq t_{0}$.

Theorem 4.1 [20] Let $x(t)$ be an n-dimensional Itô process on $t \geq 0$ satisfying the following stochastic differential equation

$d x(t)=h(t, x(t)) d t+f(t, x(t)) d B_{t}$,

where $h \in \mathcal{L}^{1}\left(\mathbb{R}_{+} ; \mathbb{R}^{n}\right)$ and $f \in \mathcal{L}^{2}\left(\mathbb{R}_{+} ; \mathbb{R}^{n \times m}\right)$.

Let $V \in C^{1,2}\left(\mathbb{R}_{+} \times \mathbb{R}^{n}, \mathbb{R}\right)$ the family of all nonnegative functions $V(t, x(t))$ defined on $\mathbb{R}_{+} \times \mathbb{R}^{n}$ which are once continuously differentiable with respect to $t$ and twice with respect to $x$.

Then, $V(t, x(t))$ is an Itô process and

$$
\begin{aligned}
& d V(t, x(t)) \\
& \quad=\mathcal{L} V(t, x(t)) d t+V_{x}(t, x(t)) f(t, x(t)) d B_{t},
\end{aligned}
$$

where

$$
\begin{aligned}
\mathcal{L} V(t, x):= & V_{t}(t, x)+V_{x}(t, x) h(t, x) \\
+ & \frac{1}{2} \operatorname{trace}\left[f^{T}(t, x) V_{x x}(t, x) f(t, x)\right], \\
V_{t}(t, x)= & \frac{\partial V}{\partial t}(t, x) ; V_{x}(t, x)=\left(\frac{\partial V}{\partial x_{1}}(t, x),\right. \\
& \left.\frac{\partial V}{\partial x_{2}}(t, x), \ldots, \frac{\partial V}{\partial x_{n}}(t, x)\right) ; \\
V_{x x}(t, x)= & \left(\frac{\partial^{2} V}{\partial x_{i} \partial x_{j}}(t, x)\right)_{n \times n} .
\end{aligned}
$$

Our approach structured in this section is to use the Lyapunov function (4.2) for the deterministic linear timevarying singular system (2.1) as a Lyapunov function candidate for the stochastic perturbed singular system (3.1) under some assumptions in the perturbation term.

Now, we state and prove one of our main results in this paper.

Theorem 4.2 Consider the stochastic perturbed singular system (3.1). Assume that there exists $P \in$ $\mathbb{C}\left(\mathbb{R}_{+}, \mathbb{R}^{n \times n}\right)$ being the solution of the generalized time varying Lyapunov equation (4.3), with $Q=Q^{T} \in$ $\mathbb{C}\left(\mathbb{R}_{+}, \mathbb{R}^{n \times n}\right)$, such that

$$
\begin{aligned}
& \exists q_{1}, q_{2}>0: \\
& q_{1} x^{T} x \leq x^{T} Q(t) x \leq q_{2} x^{T} x, \quad \forall(t, x) \in \mathcal{W},
\end{aligned}
$$


and $E^{T} P E \in \mathbb{C}^{1}\left(\mathbb{R}_{+}, \mathbb{R}^{n \times n}\right)$, such that

$$
\begin{aligned}
\exists p_{1}, p_{2} & >0: \quad p_{1} x^{T} x \leq x^{T} E^{T}(t) P(t) E(t) x \\
& \leq p_{2} x^{T} x, \forall(t, x) \in \mathcal{W} .
\end{aligned}
$$

Additionally, assume that

$$
\begin{aligned}
\|\Pi(t)\| & \leq M, \quad \forall t \geq 0, \\
\|f(t, x)\| & \leq \mu\|x\|, \quad \forall(t, x) \in \mathcal{W},
\end{aligned}
$$

where $\mu$ and $M$ are positive constants, and the matrix $Q$ is chosen such that $q_{1}>p_{2} \mu^{2} M^{2}$.

Then, the stochastic perturbed singular system (3.1) is uniformly exponentially stable in mean square.

Now, we recall the following Gronwall lemma, which will be crucial in our analysis.

Lemma 4.3 [22] Let $\varphi: \mathbb{R}_{+} \longrightarrow \mathbb{R}_{+}$be a continuous function, $\varepsilon$ is a positive real number and $\eta$ is a strictly positive real number. Assume that for all $t \in \mathbb{R}_{+}$and $0 \leq \vartheta \leq t$, we have

$\varphi(t)-\varphi(\vartheta) \leq \int_{\vartheta}^{t}(-\eta \varphi(s)+\varepsilon) d s$.

Then,

$\varphi(t) \leq \frac{\varepsilon}{\eta}+\varphi(0) \exp (-\eta t)$

Proof of Theorem 4.2. Consider the following Lyapunov like function:

$V: \mathcal{W} \rightarrow \mathbb{R}, \quad(t, x) \mapsto(E(t) x)^{T} P(t)(E(t) x)$.

Applying Itô's formula to $V(\cdot, x(\cdot))$ where $x(\cdot)$ is a solution to system (3.1), we obtain

$$
\begin{aligned}
\mathcal{L} V(t, x(t))= & x^{T}(t)\left(A^{T}(t) P(t) E(t)+\frac{d}{d t}\left(E^{T}(t) P(t) E(t)\right)\right. \\
& \left.+E^{T}(t) P(t) A(t)\right) x(t) \\
& +(E(t) \Pi(t) f(t, x(t)))^{T} \\
& P(t)(E(t) \Pi(t) f(t, x(t))) \\
= & -x^{T}(t) Q(t) x(t)+(E(t) \Pi(t) f(t, x(t)))^{T} \\
& P(t)(E(t) \Pi(t) f(t, x(t))) .
\end{aligned}
$$

One can deduce from the standing assumptions that

$$
\begin{aligned}
\mathcal{L} V(t, x(t)) & \leq-q_{1}\|x(t)\|^{2}+p_{2} \mu^{2} M^{2}\|x(t)\|^{2} \\
& =-\left(q_{1}-p_{2} \mu^{2} M^{2}\right) x^{T}(t) x(t) .
\end{aligned}
$$

Since $q_{1}>p_{2} \mu^{2} M^{2}$, then from (4.6) we obtain

$$
\mathcal{L} V(t, x(t)) \leq-\frac{q_{1}-p_{2} \mu^{2} M^{2}}{p_{2}} V(t, x(t)) .
$$

Thanks to Dynkin's formula [18], we obtain

$\mathbb{E}(V(t, x(t)))-V(0, x(0))=\int_{0}^{t} \mathbb{E}(L V(s, x(s))) d s$.

Then, for all $\vartheta, t$ with $0 \leq \vartheta \leq t \leq \infty$, it yields that

$$
\begin{aligned}
0 \leq & \mathbb{E}(V(t, x(t)))-\mathbb{E}(V(\vartheta, x(\vartheta))) \\
& \leq \int_{\vartheta}^{t} \mathbb{E}(L V(s, x(s))) d s \\
& \leq \int_{\vartheta}^{t}-\frac{q_{1}-p_{2} \mu^{2} M^{2}}{p_{2}} \mathbb{E}(V(s, x(s))) d s .
\end{aligned}
$$

Thanks now to Gronwall's lemma (Lemma 4.3), it follows that

$\mathbb{E}(V(t, x(t)))$

$$
\leq \mathbb{E}(V(0, x(0))) \exp \left(-\left(\frac{q_{1}}{p_{2}}-\mu^{2} M^{2}\right) t\right) .
$$

Next, we aim to derive an estimate for $\mathbb{E}\left(\|x(t)\|^{2}\right)$.

$$
\begin{aligned}
\mathbb{E}\left(\|x(t)\|^{2}\right) \leq & \frac{1}{p_{1}} \mathbb{E}\left((E(t) x(t))^{T} P(t)(E(t) x(t))\right) \\
& \leq \frac{1}{p_{1}} \mathbb{E}(V(0, x(0))) \\
& \times \exp \left(-\left(\frac{q_{1}}{p_{2}}-\mu^{2} M^{2}\right) t\right) \\
& =\frac{1}{p_{1}} x^{T}(0) E^{T}(0) P(0) E(0) x(0) \exp \\
& \left(-\left(\frac{q_{1}}{p_{2}}-\mu^{2} M^{2}\right) t\right) \\
& \leq \frac{p_{2}}{p_{1}}\left\|x_{0}\right\|^{2} \exp \left(-\left(\frac{q_{1}}{p_{2}}-\mu^{2} M^{2}\right) t\right) .
\end{aligned}
$$


That is,

$\mathbb{E}\left(\|x(t)\|^{2}\right) \leq \frac{p_{2}}{p_{1}}\left\|x_{0}\right\|^{2} \exp \left(-\left(\frac{q_{1}}{p_{2}}-\mu^{2} M^{2}\right) t\right)$.

Hence, the stochastic perturbed singular system (3.1) is uniformly exponentially stable in mean square.

\subsection{Practical exponential stability of the stochastic} perturbed singular system in mean square

In this section, we study the exponential stability in mean square of a nontrivial solution of the stochastic perturbed singular system (3.1). We suppose that $f(t, 0)$ is not necessarily zero.

Now, we define the exponential stability in mean square for the stochastic perturbed singular system (3.1) when the origin is no longer an equilibrium point. In this case, we study the stability of solutions with respect to a small neighborhood of the origin.

The study of the asymptotic behavior of solutions leads to investigate the stability behavior of a small ball centered at the origin: $B_{r}:=\left\{x \in \mathbb{R}^{n} \mid\|x\| \leq r\right\}, r>$ 0 .

Definition 4.2 i) $B_{r}$ is uniformly exponentially stable in mean square, if there exist positive constants $\lambda_{1}$ and $\lambda_{2}$, such that for all $\left(t_{0}, x_{0}\right) \in \mathcal{W}$,

$\mathbb{E}\left(\|x(t)\|^{2}\right) \leq \lambda_{1}\left\|x_{0}\right\|^{2} \exp \left(-\lambda_{2}\left(t-t_{0}\right)\right)+r, \quad \forall t \geq t_{0}$.

ii) The stochastic perturbed singular system (3.1) is said to be practically uniformly exponentially stable in mean square, if there exists $r>0$, such that $B_{r}$ is uniformly exponentially stable in mean square.

Now, we proceed to investigate the practical exponential stability in mean square of stochastic perturbed singular system (3.1) by using Lyapunov functions.

Theorem 4.4 Consider the stochastic perturbed singular system (3.1). Assume that there exists $P \in$ $\mathbb{C}\left(\mathbb{R}_{+}, \mathbb{R}^{n \times n}\right)$ being the solution of the generalized time varying Lyapunov equation (4.3), with $Q=$ $Q^{T} \in \mathbb{C}\left(\mathbb{R}_{+}, \mathbb{R}^{n \times n}\right)$ satisfying condition (4.5), and $E^{T} P E \in \mathbb{C}^{1}\left(\mathbb{R}_{+}, \mathbb{R}^{n \times n}\right)$ fulfills condition (4.6). Furthermore, suppose that $\Pi$ satisfies (4.7), and $f(t, x)$ satisfies the following condition:

$\|f(t, x)\|^{2} \leq \gamma\|x\|^{2}+\phi(t), \quad \forall(t, x) \in \mathcal{W}$, where $\phi(\cdot)$ is a nonnegative continuous bounded function. Assume that the matrix $Q$ is chosen such that $q_{1}>p_{2} M^{2} \gamma$. Then, the stochastic perturbed singular system (3.1) is practically uniformly exponentially stable in mean square.

Proof Consider the following Lyapunov function:

$V: \mathcal{W} \rightarrow \mathbb{R}, \quad(t, x) \mapsto(E(t) x)^{T} P(t)(E(t) x)$.

By the Itô formula applied to $V(\cdot, x(\cdot))$, where $x(\cdot)$ is a solution to (3.1), we obtain

$$
\begin{aligned}
\mathcal{L} V(t, x(t))= & x^{T}(t)\left(A^{T}(t) P(t) E(t)+\frac{d}{d t}\left(E^{T}(t) P(t) E(t)\right)\right. \\
& \left.+E^{T}(t) P(t) A(t)\right) x(t) \\
& +(E(t) \Pi(t) f(t, x(t)))^{T} \\
& P(t)(E(t) \Pi(t) f(t, x(t))) \\
= & -x^{T}(t) Q(t) x(t)+(E(t) \Pi(t) f(t, x(t)))^{T} \\
& P(t)(E(t) \Pi(t) f(t, x(t))) .
\end{aligned}
$$

Taking into account the assumptions, we obtain

$$
\begin{aligned}
\mathcal{L} V & (t, x(t)) \\
& \leq-q_{1} x^{T}(t) x(t)+p_{2} M^{2} \gamma x^{T}(t) x(t)+p_{2} M^{2} \phi(t) \\
& =-\left(q_{1}-p_{2} M^{2} \gamma\right) x^{T}(t) x(t)+p_{2} M^{2} \phi(t) .
\end{aligned}
$$

Since $q_{1}>p_{2} M^{2} \gamma$, we deduce that

$\mathcal{L} V(t, x(t)) \leq-\frac{q_{1}-p_{2} M^{2} \gamma}{p_{2}} V(t, x(t))+p_{2} M^{2} \phi(t)$.

On the other side, recall that $t \mapsto \phi(t)$ is nonnegative and bounded on $\mathbb{R}_{+}$. Therefore, there exists $m>0$, such that

$\phi(t) \leq m$, for all $t \geq 0$.

Consequently, we obtain

$$
\begin{aligned}
\mathcal{L} V(t, x(t)) & \leq-\frac{q_{1}-p_{2} M^{2} \gamma}{p_{2}} V(t, x(t))+p_{2} m M^{2} \\
& =-\left[\frac{q_{1}}{p_{2}}-M^{2} \gamma\right] V(t, x(t))+p_{2} m M^{2} .
\end{aligned}
$$


Dynkin's formula [18] yields that

$\mathbb{E}(V(t, x(t)))-V(0, x(0))=\int_{0}^{t} \mathbb{E}(L V(s, x(s))) d s$.

Thus, for all $\vartheta, t$ such that $0 \leq \vartheta \leq t \leq \infty$, it follows that

$$
\begin{aligned}
0 & \leq \mathbb{E}(V(t, x(t)))-\mathbb{E}(V(\vartheta, x(\vartheta))) \\
& \leq \int_{\vartheta}^{t} \mathbb{E}(L V(s, x(s))) d s \\
& \leq \int_{\vartheta}^{t}-\left[\frac{q_{1}}{p_{2}}-M^{2} \gamma\right] \mathbb{E}(V(s, x(s)))+p_{2} m M^{2} d s .
\end{aligned}
$$

Using once more the Gronwall lemma (Lemma 4.3), the previous inequality implies

$$
\begin{aligned}
& \mathbb{E}(V(t, x(t))) \\
& \leq \mathbb{E}(V(0, x(0))) \exp \left(-\left(\frac{q_{1}}{p_{2}}-M^{2} \gamma\right) t\right) \\
& \quad+\frac{p_{2}^{2} m M^{2}}{q_{1}-p_{2} M^{2} \gamma} .
\end{aligned}
$$

Now, we can estimate the term $\mathbb{E}\left(\|x(t)\|^{2}\right)$ as follows:

$$
\begin{aligned}
\mathbb{E}\left(\|x(t)\|^{2}\right) \leq & \frac{1}{p_{1}} \mathbb{E}\left((E(t) x(t))^{T} P(t)(E(t) x(t))\right) \\
\leq & \frac{1}{p_{1}} \mathbb{E}(V(0, x(0))) \exp \left(-\left(\frac{q_{1}}{p_{2}}-M^{2} \gamma\right) t\right) \\
& +\frac{p_{2}^{2} m M^{2}}{p_{1}\left(q_{1}-p_{2} M^{2} \gamma\right)} \\
= & \frac{1}{p_{1}} x^{T}(0) E^{T}(0) P(0) E(0) x(0) \\
& \exp \left(-\left(\frac{q_{1}}{p_{2}}-M^{2} \gamma\right) t\right)+\frac{p_{2}^{2} m M^{2}}{p_{1}\left(q_{1}-p_{2} M^{2} \gamma\right)} \\
\leq & \frac{p_{2}}{p_{1}}\left\|x_{0}\right\|^{2} \exp \left(-\left(\frac{q_{1}}{p_{2}}-M^{2} \gamma\right) t\right) \\
& +\frac{p_{2}^{2} m M^{2}}{p_{1}\left(q_{1}-p_{2} M^{2} \gamma\right)} .
\end{aligned}
$$

Thus, we conclude that for all $\left(0, x_{0}\right) \in \mathcal{W}$,

$$
\begin{aligned}
\mathbb{E}\left(\|x(t)\|^{2}\right) \leq & \frac{p_{2}}{p_{1}}\left\|x_{0}\right\|^{2} \exp \left(-\left(\frac{q_{1}}{p_{2}}-M^{2} \gamma\right) t\right) \\
& +\frac{p_{2}^{2} m M^{2}}{p_{1}\left(q_{1}-p_{2} M^{2} \gamma\right)} .
\end{aligned}
$$

Setting, $\lambda_{1}=\frac{p_{2}}{p_{1}}, \quad \lambda_{2}=\left(\frac{q_{1}}{p_{2}}-M^{2} \gamma\right), \quad$ and $r=\frac{p_{2}^{2} m M^{2}}{p_{1}\left(q_{1}-p_{2} M^{2} \gamma\right)}$, we deduce that the stochastic perturbed singular system (3.1) is practically uniformly exponentially stable in mean square.

Both theorems 4.2 and 4.4 show that the solution $P(\cdot)$ of (4.3) is not the target of interest, but $E^{T}(\cdot) P(\cdot) E(\cdot)$. Symmetry, differentiability, and the boundary conditions are not deserving for $P(\cdot)$, but for $E^{T}(\cdot) P(\cdot) E(\cdot)$. In the remainder of this section, we aim to derive an extra requirement upon $E(\cdot)$ under which one might obtain a sequel, where all conditions are made on $P(\cdot)$. This means that our target in the next lemma is to state the relationship between $P(\cdot)$ and $E^{T}(\cdot) P(\cdot) E(\cdot)$.

To this end, we add the following notation:

$\xi \mathcal{W}:=\left\{(t, x) \in \mathbb{R}_{+} \times \mathbb{R}^{n}: x \in E(t) \mathcal{W}(t)\right\}$.

Lemma 4.5 Consider the stochastic perturbed singular system (3.1), and let $E^{T} E \in \mathbb{C}\left(\mathbb{R}_{+}, \mathbb{R}^{n \times n}\right)$, such that

$\exists e_{1}, e_{2}>0: \quad e_{1} x^{T} x \leq x^{T} E^{T}(t) E(t) x \leq e_{2} x^{T} x$,

$$
\forall(t, x) \in \mathcal{W} \text {. }
$$

Let also $P \in \mathbb{C}\left(\mathbb{R}_{+}, \mathbb{R}^{n \times n}\right)$ be a symmetric matrix.

Then,

$\exists \bar{p}_{1}, \bar{p}_{2}>0: \quad \bar{p}_{1} x^{T} x \leq x^{T} P(t) x \leq \bar{p}_{2} x^{T} x$,

$$
\forall(t, x) \in \xi \mathcal{W},
$$

if and only if,

$$
\begin{gathered}
\exists p_{1}, p_{2}>0: p_{1} x^{T} x \leq x^{T} E^{T}(t) P(t) E(t) x \leq p_{2} x^{T} x, \\
\forall(t, x) \in \mathcal{W} .
\end{gathered}
$$

Proof " $\Rightarrow$ " Let $(t, x) \in \mathcal{W}$, that is $E(t) x \in$ $E(t) \mathcal{W}(t)$.

Based upon (4.10) and (4.11), it follows that

$$
\begin{aligned}
\bar{p}_{1} e_{1} x^{T} x & \leq \bar{p}_{1} x^{T} E^{T}(t) E(t) x \leq x^{T} E^{T}(t) P(t) E(t) x \\
& \leq \bar{p}_{2} x^{T} E^{T}(t) E(t) x \leq \bar{p}_{1} e_{2} x^{T} x .
\end{aligned}
$$


Hence, for $p_{1}=\bar{p}_{1} e_{1}$ and $p_{2}=\bar{p}_{2} e_{2}$ condition (4.12) holds.

" $\Leftarrow "$ Let $(t, x) \in \xi \mathcal{W}$, so $x \in E(t) \mathcal{W}(t)$ and therefore there exists $z \in \mathcal{W}(t)$, such that $x=E(t) z$.

Taking into account (4.12) and (4.10), one obtains

$$
\begin{aligned}
\frac{p_{1}}{e_{2}} x^{T} x & \leq \frac{p_{1}}{e_{2}}(E(t) z)^{T}(E(t) z) \leq p_{1} z^{T} z \\
& \leq z^{T} E^{T}(t) P(t) E(t) z
\end{aligned}
$$

\section{Practical exponential stability in mean square of a class of stochastic singular systems with uncertainties}

In this section, we discuss the problem of stabilization for a class of stochastic singular systems with uncertainties.

Consider the following system:

$\left\{\begin{aligned} E(t) \dot{x}(t) & =A(t) x(t)+H(t)(\Psi(t, x, u)+u)+E(t) \Pi(t) f(t, x(t)) d B_{t}, \\ x(0) & =x_{0},\end{aligned}\right.$

$$
\begin{aligned}
& =x^{T} P(t) x \leq p_{2} z^{T} z \\
& \leq \frac{p_{2}}{e_{1}}(E(t) z)^{T}(E(t) z)=\frac{p_{2}}{e_{1}} x^{T} x .
\end{aligned}
$$

Thus, we have

$$
\frac{p_{1}}{e_{2}} x^{T} x \leq x^{T} P(t) x \leq \frac{p_{2}}{e_{1}} x^{T} x, \quad \forall(t, x) \in \xi \mathcal{W} .
$$

Setting, $\bar{p}_{1}=\frac{p_{1}}{e_{2}}$ and $\bar{p}_{2}=\frac{p_{2}}{e_{1}}$, condition (4.11) holds.

Now, we are ready to state the following corollary.

Corollary 4.6 Consider the stochastic perturbed singular system (3.1).

Assume $E \in \mathbb{C}^{1}\left(\mathbb{R}_{+}, \mathbb{R}^{n \times n}\right)$ and satisfies (4.10). Suppose that there exist $Q=Q^{T} \in \mathbb{C}\left(\mathbb{R}_{+}, \mathbb{R}^{n \times n}\right)$ satisfying (4.5) and $P \in \mathbb{C}\left(\mathbb{R}_{+}, \mathbb{R}^{n \times n}\right)$, fulfilling (4.11), such that (4.3) holds. In addition, we assume $\Pi$ satisfies (4.7), $f(t, x)$ satisfies condition (4.8), and the matrix $Q$ is chosen such that $q_{1}>p_{2} \mu^{2} M^{2}$.

Then, the stochastic perturbed singular system (3.1) is uniformly exponentially stable in mean square.

Proof The proof is straightforward. Owing to Lemma 4.5, we have $E^{T} P E \in \mathbb{C}^{1}\left(\mathbb{R}_{+}, \mathbb{R}^{n \times n}\right)$ and satisfies condition (4.12). Hence, all conditions of Theorem 4.2 are fulfilled and the stochastic perturbed singular system (3.1) is uniformly exponentially stable in mean square.

Remark 4.7 Under the same assumptions of Corollary 4.6 with $f(t, x)$ satisfying condition (4.9), the stochastic perturbed singular system (3.1) is practically uniformly exponentially stable in mean square. where $x(t) \in \mathbb{R}^{n}$ represents the state, $u \in \mathbb{R}^{m}$ is the control input, $B_{t} \in \mathbb{R}$ is a standard Brownian motion defined on a complete probability space $(\Omega, F, P)$ with $B_{0}=0 .(E, A) \in \mathbb{C}\left(\mathbb{R}_{+}, \mathbb{R}^{n \times n}\right)^{2}$ with $E$ being a singular matrix. $\Pi \in \mathbb{C}\left(\mathbb{R}_{+}, \mathbb{R}^{n \times n}\right)$, such that $\operatorname{im} \Pi(t)=$ $\mathcal{W}(t)$ for all $t \in \mathbb{R}_{+}, H(t) \in \mathbb{C}\left(\mathbb{R}_{+}, \mathbb{R}^{n \times m}\right)$, and the function $\Psi: \mathbb{R}_{+} \times \mathbb{R}^{n} \times \mathbb{R}^{m} \rightarrow \mathbb{R}^{m}$ represents uncertainties in the plant. Assume that the pair $(E, A)$ is transferable into a standard canonical form, and $f: \mathbb{R}_{+} \times \mathbb{R}^{n} \rightarrow \mathbb{R}^{n}$ satisfies both conditions (3.2) and (3.3).

Let us state now some assumptions which will be imposed later on:

$\left(H_{1}\right)$ There exists a symmetric matrix $P \in \mathbb{C}\left(\mathbb{R}_{+}, \mathbb{R}^{n \times n}\right)$ being the solution of the generalized timevarying Lyapunov equation (4.3), with $Q=$ $Q^{T} \in \mathbb{C}\left(\mathbb{R}_{+}, \mathbb{R}^{n \times n}\right)$ satisfying condition (4.5).

( $\left.H_{2}\right) E^{T} P E \in \mathbb{C}^{1}\left(\mathbb{R}_{+}, \mathbb{R}^{n \times n}\right)$ fulfills condition (4.6).

$\left(H_{3}\right)$ There exists a nonnegative continuous function $\Phi: \mathbb{R}^{n} \rightarrow \mathbb{R}_{+}$, such that for all $t \in \mathbb{R}_{+}, x \in \mathbb{R}^{n}, u \in \mathbb{R}^{m}$ :

$$
\|\Psi(t, x, u)\| \leq \Phi(x)
$$

$\left(H_{4}\right) \Pi$ satisfies (4.7) and there exists a positive constant $\bar{\mu}$, such that

$$
\|f(t, x)\| \leq \bar{\mu}\|x\|, \text { a.s. } \forall(t, x) \in \mathcal{W} .
$$

Furthermore, we assume that $Q$ is chosen such that $q_{1}>p_{2} M^{2} \bar{\mu}^{2}$. 
Theorem 5.1 Suppose that assumptions $\left(H_{1}\right)-\left(H_{4}\right)$ hold, then the feedback law

$u(t, x)=-\frac{H^{T}(t) P(t) E(t) x \Phi^{2}(x)}{\left\|H^{T}(t) P(t) E(t) x\right\| \Phi(x)+\rho(t)}$,

where $\rho(t)$ is a continuous nonnegative bounded function, uniformly practically exponentially stabilizes system (5.1) in mean square.

Proof Consider the following Lyapunov-like function:

$$
V: \mathcal{W} \rightarrow \mathbb{R}, \quad(t, x) \mapsto(E(t) x)^{T} P(t)(E(t) x) .
$$

Invoking Itô's formula to $V(\cdot)$ along the trajectory $x(\cdot)$ of the stochastic singular system (5.1), we obtain

$$
\begin{aligned}
& \mathcal{L} V(t, x(t)) \\
&=x^{T}(t)\left(A^{T}(t) P(t) E(t)+\frac{d}{d t}\left(E^{T}(t) P(t) E(t)\right)\right. \\
&\left.+E^{T}(t) P(t) A(t)\right) x(t) \\
&+2 x^{T}(t) E^{T}(t) P(t) H(t) \Psi(t, x, u) \\
&+(E(t) \Pi(t) f(t, x(t)))^{T} P(t)(E(t) \Pi(t) f(t, x(t))) \\
&=-x^{T}(t) Q(t) x(t) \\
&-\frac{2 x^{T}(t) E^{T}(t) P(t) H(t) H^{T}(t) P(t) E(t) x(t) \Phi^{2}(x(t))}{\left\|H^{T}(t) P(t) E(t) x(t)\right\| \Phi(x(t))+\rho(t)} \\
&+2 x^{T}(t) E^{T}(t) P(t) H(t) \Psi(t, x, u) \\
&+(E(t) \Pi(t) f(t, x(t)))^{T} P(t)(E(t) \Pi(t) f(t, x(t))) .
\end{aligned}
$$

Assumption $\left(H_{3}\right)$ implies

$$
\begin{aligned}
& \mathcal{L} V(t, x(t)) \\
& \leq-x^{T}(t) Q(t) x(t) \\
&-\frac{2 x^{T}(t) E^{T}(t) P(t) H(t) H^{T}(t) P(t) E(t) x(t) \Phi^{2}(x(t))}{\left\|H^{T}(t) P(t) E(t) x(t)\right\| \Phi(x(t))+\rho(t)} \\
&+2\left\|H^{T}(t) P(t) E(t) x(t)\right\| \Phi(x(t)) \\
&+(E(t) \Pi(t) f(t, x(t)))^{T} P(t)(E(t) \Pi(t) f(t, x(t))) \\
& \leq-x^{T}(t) Q(t) x(t) \\
&+\frac{2\left\|H^{T}(t) P(t) E(t) x(t)\right\| \Phi(x(t)) \rho(t)}{\left\|H^{T}(t) P(t) E(t) x(t)\right\| \Phi(x(t))+\rho(t)} \\
&+(E(t) \Pi(t) f(t, x(t)))^{T} P(t)(E(t) \Pi(t) f(t, x(t))) .
\end{aligned}
$$

Owing to the following inequality:

$$
\frac{\left\|H^{T}(t) P(t) E(t) x(t)\right\| \Phi(x(t)) \rho(t)}{\left\|H^{T}(t) P(t) E(t) x(t)\right\| \Phi(x(t))+\rho(t)} \leq \rho(t) .
$$

Thus, we obtain

$$
\begin{aligned}
\mathcal{L} V(t, x(t)) \leq & -x^{T}(t) Q(t) x(t)+2 \rho(t) \\
+ & (E(t) \Pi(t) f(t, x(t)))^{T} \\
& P(t)(E(t) \Pi(t) f(t, x(t))) .
\end{aligned}
$$

Since $\rho(t)$ is a continuous nonnegative bounded function, then there exists $\bar{\rho}>0$, such that

$\|\rho(t)\| \leq \bar{\rho}, \quad \forall t \geq 0$.

Based on the assumptions, it follows that

$\mathcal{L} V(t, x(t)) \leq-\left(q_{1}-p_{2} M^{2} \bar{\mu}^{2}\right) x^{T}(t) x(t)+2 \bar{\rho}$.

As $q_{1}>p_{2} M^{2} \bar{\mu}^{2}$, assumption $\left(H_{1}\right)$ implies that

$\mathcal{L} V(t, x(t)) \leq-\frac{q_{1}-p_{2} M^{2} \bar{\mu}^{2}}{p_{2}} V(t, x(t))+2 \bar{\rho}$.

Thanks to Dynkin's formula [18], it follows that

$\mathbb{E}(V(t, x(t)))-V(0, x(0))=\int_{0}^{t} \mathbb{E}(L V(s, x(s))) d s$.

Thus, for all $\vartheta, t$ such that $0 \leq \vartheta \leq t \leq \infty$, we obtain

$$
\begin{aligned}
0 & \leq \mathbb{E}(V(t, x(t)))-\mathbb{E}(V(\vartheta, x(\vartheta))) \\
& \leq \int_{\vartheta}^{t} \mathbb{E}(L V(s, x(s))) d s \\
& \leq \int_{\vartheta}^{t}-\left[\frac{q_{1}}{p_{2}}-M^{2} \bar{\mu}^{2}\right] \mathbb{E}(V(s, x(s))) d s+2 \bar{\rho} .
\end{aligned}
$$

By applying the Gronwall Lemma 4.3,

$$
\begin{aligned}
& \mathbb{E}(V(t, x(t))) \\
& \leq \mathbb{E}(V(0, x(0))) \exp \left(-\left(\frac{q_{1}}{p_{2}}-M^{2} \bar{\mu}^{2}\right) t\right) \\
& \quad+\frac{2 \bar{\rho} p_{2}}{q_{1}-p_{2} M^{2} \bar{\mu}^{2}} .
\end{aligned}
$$


Now, we aim to derive an estimate for $\mathbb{E}\left(\|x(t)\|^{2}\right)$,

$$
\begin{aligned}
& \mathbb{E}\left(\|x(t)\|^{2}\right) \\
& \leq \frac{1}{p_{1}} \mathbb{E}\left((E(t) x(t))^{T} P(t)(E(t) x(t))\right) \\
& \leq \frac{1}{p_{1}} \mathbb{E}(V(0, x(0))) \exp \left(-\left(\frac{q_{1}}{p_{2}}-M^{2} \bar{\mu}^{2}\right) t\right) \\
&+\frac{2 \bar{\rho} p_{2}}{p_{1}\left(q_{1}-p_{2} M^{2} \bar{\mu}^{2}\right)} \\
&= \frac{1}{p_{1}} x^{T}(0) E^{T}(0) P(0) E(0) x(0) \\
& \times \exp \left(-\left(\frac{q_{1}}{p_{2}}-M^{2} \bar{\mu}^{2}\right) t\right)+\frac{2 \bar{\rho} p_{2}}{p_{1}\left(q_{1}-p_{2} M^{2} \bar{\mu}^{2}\right)} \\
& \leq \frac{p_{2}}{p_{1}}\left\|x_{0}\right\|^{2} \exp \left(-\left(\frac{q_{1}}{p_{2}}-M^{2} \bar{\mu}^{2}\right) t\right) \\
&+\frac{2 \bar{\rho} p_{2}}{p_{1}\left(q_{1}-p_{2} M^{2} \bar{\mu}^{2}\right)} .
\end{aligned}
$$

Indeed, we have derived that for all $\left(0, x_{0}\right) \in \mathcal{W}$,

$$
\begin{aligned}
\mathbb{E}\left(\|x(t)\|^{2}\right) \leq & \frac{p_{2}}{p_{1}}\left\|x_{0}\right\|^{2} \exp \left(-\left(\frac{q_{1}}{p_{2}}-M^{2} \bar{\mu}^{2}\right) t\right) \\
& +\frac{2 \bar{\rho} p_{2}}{p_{1}\left(q_{1}-p_{2} M^{2} \bar{\mu}^{2}\right)} .
\end{aligned}
$$

Hence, we conclude that the uncertain closed-loop stochastic singular system (5.1) is uniformly practically exponentially stable in mean square.

Corollary 5.2 If we consider the special case with $\rho(t)=\frac{\alpha}{2} e^{-\gamma t}$, where $\alpha>0$ and $\gamma>0$, one obtains that the uncertain closed-loop stochastic singular system (5.1) is uniformly exponentially stable in mean square, such that for all $\left(0, x_{0}\right) \in \mathcal{W}$, we have

$$
\begin{aligned}
& \mathbb{E}\left(\|x(t)\|^{2}\right) \\
& \leq\left\{\begin{array}{l}
\frac{p_{2}}{p_{1}}\left\|x_{0}\right\|^{2} e^{-\tilde{\lambda} t}+\frac{\alpha}{p_{1}} t e^{-\tilde{\lambda} t}, \quad \text { if } \gamma=\tilde{\lambda}, \\
\frac{p_{2}}{p_{1}}\left\|x_{0}\right\|^{2} e^{-\tilde{\lambda} t}+\frac{\alpha}{p_{1}(\tilde{\lambda}-\gamma)}\left(e^{-\gamma t}-e^{-\tilde{\lambda} t}\right), \quad \text { if } \gamma \neq \tilde{\lambda},
\end{array}\right.
\end{aligned}
$$

where $\tilde{\lambda}=\frac{q_{1}-p_{2} M^{2} \bar{\mu}^{2}}{p_{2}}$.

Proof Consider the following Lyapunov-like function:

$$
V: \mathcal{W} \rightarrow \mathbb{R}, \quad(t, x) \mapsto(E(t) x)^{T} P(t)(E(t) x) .
$$

Based on Theorem 5.1 we have

$$
\mathcal{L} V(t, x(t)) \leq-\tilde{\lambda} V(t, x(t))+2 \rho(t),
$$

where $\tilde{\lambda}=\frac{q_{1}-p_{2} M^{2} \bar{\mu}^{2}}{p_{2}}$. In particular, for $\rho(t)=$ $\frac{\alpha}{2} e^{-\gamma t}$ one obtains

$\mathcal{L} V(t, x(t)) \leq-\tilde{\lambda} V(t, x(t))+\alpha e^{-\gamma t}$.

Now, for any $B>0$, we define the following stopping time:

$\tau_{B}=\inf \{t \geq 0:\|x(t)\| \geq B\}$.

Obviously $\tau_{B} \rightarrow \infty$, as $B \rightarrow \infty$ almost surely. By Itô's formula, it follows that for $t \geq 0$

$$
\begin{aligned}
& \mathbb{E}\left(e^{\tilde{\lambda}\left(t \wedge \tau_{B}\right)} V\left(t \wedge \tau_{B}, x\left(t \wedge \tau_{B}\right)\right)\right) \\
&= V(0, x(0))+\mathbb{E}\left(\int_{0}^{t \wedge \tau_{B}} e^{\tilde{\lambda} s}[\tilde{\lambda} V(s, x(s))\right. \\
&+\mathcal{L} V(s, x(s))] d s) \\
& \leq V(0, x(0))+\mathbb{E}\left(\int_{0}^{t \wedge \tau_{B}} e^{\tilde{\lambda} s}(\tilde{\lambda} V(s, x(s))\right. \\
&\left.\left.-\tilde{\lambda} V(s, x(s))+\alpha e^{-\gamma s}\right) d s\right) \\
& \leq V(0, x(0))+\mathbb{E}\left(\int_{0}^{t \wedge \tau_{B}} \alpha e^{(\tilde{\lambda}-\gamma) s} d s\right) .
\end{aligned}
$$

That is,

$$
\begin{aligned}
& \mathbb{E}\left(e^{\tilde{\lambda}\left(t \wedge \tau_{B}\right)} V\left(t \wedge \tau_{B}, x\left(t \wedge \tau_{B}\right)\right)\right) \\
& \quad \leq V(0, x(0))+\mathbb{E}\left(\int_{0}^{t \wedge \tau_{B}} \alpha e^{(\widetilde{\lambda}-\gamma) s} d s\right) .
\end{aligned}
$$

Taking, into account assumption $\left(H_{2}\right)$, it follows that

$$
\begin{aligned}
& p_{1} \mathbb{E}\left(e^{\tilde{\lambda}\left(t \wedge \tau_{B}\right)}\left\|x\left(t \wedge \tau_{B}\right)\right\|^{2}\right) \\
& \leq V(0, x(0))+\alpha \mathbb{E}\left(\int_{0}^{t \wedge \tau_{B}} e^{(\widetilde{\lambda}-\gamma) s} d s\right) \\
& \leq p_{2}\left\|x_{0}\right\|^{2}+\alpha \mathbb{E}\left(\int_{0}^{t \wedge \tau_{B}} e^{(\widetilde{\lambda}-\gamma) s} d s\right) .
\end{aligned}
$$

Hence, we obtain

$$
\begin{aligned}
& \mathbb{E}\left(e^{\tilde{\lambda}\left(t \wedge \tau_{B}\right)}\left\|x\left(t \wedge \tau_{B}\right)\right\|^{2}\right) \\
& \leq \frac{p_{2}}{p_{1}}\left\|x_{0}\right\|^{2}+\frac{\alpha}{p_{1}} \mathbb{E}\left(\int_{0}^{t \wedge \tau_{B}} e^{(\tilde{\lambda}-\gamma) s} d s\right) .
\end{aligned}
$$

Letting $B \rightarrow \infty$ yields that

$$
\mathbb{E}\left(e^{\tilde{\lambda} t}\|x(t)\|^{2}\right) \leq \frac{p_{2}}{p_{1}}\left\|x_{0}\right\|^{2}+\frac{\alpha}{p_{1}} \int_{0}^{t} e^{(\widetilde{\lambda}-\gamma) s} d s .
$$


Thus,

$\mathbb{E}\left(\|x(t)\|^{2}\right) \leq \frac{p_{2}}{p_{1}}\left\|x_{0}\right\|^{2} e^{-\tilde{\lambda} t}+\frac{\alpha}{p_{1}} e^{-\tilde{\lambda} t} \int_{0}^{t} e^{(\tilde{\lambda}-\gamma) s} d s$.

Finally, we obtain for all $\left(0, x_{0}\right) \in \mathcal{W}$,

$\mathbb{E}\left(\|x(t)\|^{2}\right) \leq\left\{\begin{array}{l}\frac{p_{2}}{p_{1}}\left\|x_{0}\right\|^{2} e^{-\tilde{\lambda} t}+\frac{\alpha}{p_{1}} t e^{-\tilde{\lambda} t}, \quad \text { if } \gamma=\tilde{\lambda}, \\ \frac{p_{2}}{p_{1}}\left\|x_{0}\right\|^{2} e^{-\tilde{\lambda} t}+\frac{\alpha}{p_{1}(\widetilde{\lambda}-\gamma)}\left(e^{-\gamma t}-e^{-\tilde{\lambda} t}\right), \quad \text { if } \gamma \neq \tilde{\lambda} .\end{array}\right.$

\section{Example}

We provide the following illustrative examples to show the applicability of our abstract theory.

Example 6.1 Consider the following time-varying stochastic singular system:

$E(t) d x(t)=A(t) x(t) d t+E(t) \Pi(t) f(t, x(t)) d B_{t}$,

where $x=\left(x_{1}, x_{2}\right) \in \mathbb{R}^{2}, B_{t}$ is a one-dimensional Brownian motion.

$$
\begin{aligned}
E(t) & =\left(\begin{array}{ll}
1 & 0 \\
0 & 0
\end{array}\right), \quad A(t)=\left(\begin{array}{cc}
-t & 0 \\
0 & 1
\end{array}\right), \\
f(t, x) & =\left(\begin{array}{l}
f_{1}(t, x) \\
f_{2}(t, x)
\end{array}\right),
\end{aligned}
$$

with

$$
\left\{\begin{array}{l}
f_{1}(t, x)=\frac{1}{6} \frac{x_{1}^{2}}{1+\sqrt{x_{1}^{2}+x_{2}^{2}}} \\
f_{2}(t, x)=\frac{1}{6} \frac{x_{2}^{2}}{1+\sqrt{x_{1}^{2}+x_{2}^{2}}} .
\end{array}\right.
$$

System (6.1) can be regarded as a singular perturbed system of the following linear time-varying singular system:

$E(t) d x(t)=A(t) x(t) d t$.
It is clear that $(E, A)$ is transferable into a standard canonical form with $S(\cdot)=T(\cdot)=I_{2}$. Subsequently, based upon Proposition 2.1, we obtain $\mathcal{W}=\mathbb{R}_{+} \times$ $\operatorname{im}\left(\begin{array}{l}1 \\ 0\end{array}\right)$.

Let $\widetilde{p}(\cdot): \mathbb{R}_{+} \rightarrow \mathbb{R}_{+}, \quad t \mapsto e^{t^{2}} \int_{t}^{+\infty} e^{-s^{2}} d s$, and

$P(t)=\left(\begin{array}{cc}\tilde{p}(t) & 0 \\ 0 & 0\end{array}\right), \quad Q(t)=\left(\begin{array}{ll}1 & 0 \\ 0 & 1\end{array}\right)$.

One can verify that $P(\cdot)$ and $Q(\cdot)$ solve Eq. (4.3). Then, by some easy computations, we can check that $p_{2}=$ $\frac{\sqrt{\pi}}{2}, q_{1}=q_{2}=1$.

Since $\operatorname{im} \Pi(t)=\mathcal{W}(t)$, then we may choose $\Pi(t)=$ $\left(\begin{array}{ll}1 & 0 \\ 0 & 0\end{array}\right)$.

Hence, we obtain $E \Pi f=\left(\begin{array}{c}f_{1} \\ 0\end{array}\right)$, and

$\|f(t, x)\|^{2}=f_{1}^{2}(t, x)+f_{2}^{2}(t, x)$,

where $\|$.$\| represents the Euclidean norm.$

Thus, for all $(t, x) \in \mathcal{W}$, we have

$\|f(t, x)\|^{2} \leq \frac{1}{36}\|x\|^{2}$.

We can easily check that assumptions in Theorem 4.2 hold with $M=1, \mu=\frac{1}{6}$.

Clearly, $q_{1}>p_{2} \mu^{2} M^{2}$. Finally, we deduce that the stochastic perturbed singular system (6.1) is uniformly exponentially stable in mean square, as shown in Fig. 1.

Example 6.2 Consider the following time-varying stochastic singular system:

$E(t) d x(t)=A(t) x(t) d t+E(t) \Pi(t) f(t, x(t)) d B_{t}$,

where $x=\left(x_{1}, x_{2}\right) \in \mathbb{R}^{2}, B_{t}$ is a one-dimensional Brownian motion.

$$
\begin{aligned}
E(t) & =\left(\begin{array}{ll}
1 & 0 \\
0 & 0
\end{array}\right), \quad A(t)=\left(\begin{array}{cc}
-1 & 0 \\
0 & e^{t}
\end{array}\right), \\
f(t, x) & =\left(\begin{array}{l}
f_{1}(t, x) \\
f_{2}(t, x)
\end{array}\right),
\end{aligned}
$$


Fig. 1 The initial response of the system 6.1, with five different Brownian motions, and $x_{0}=[1,0]^{T}$

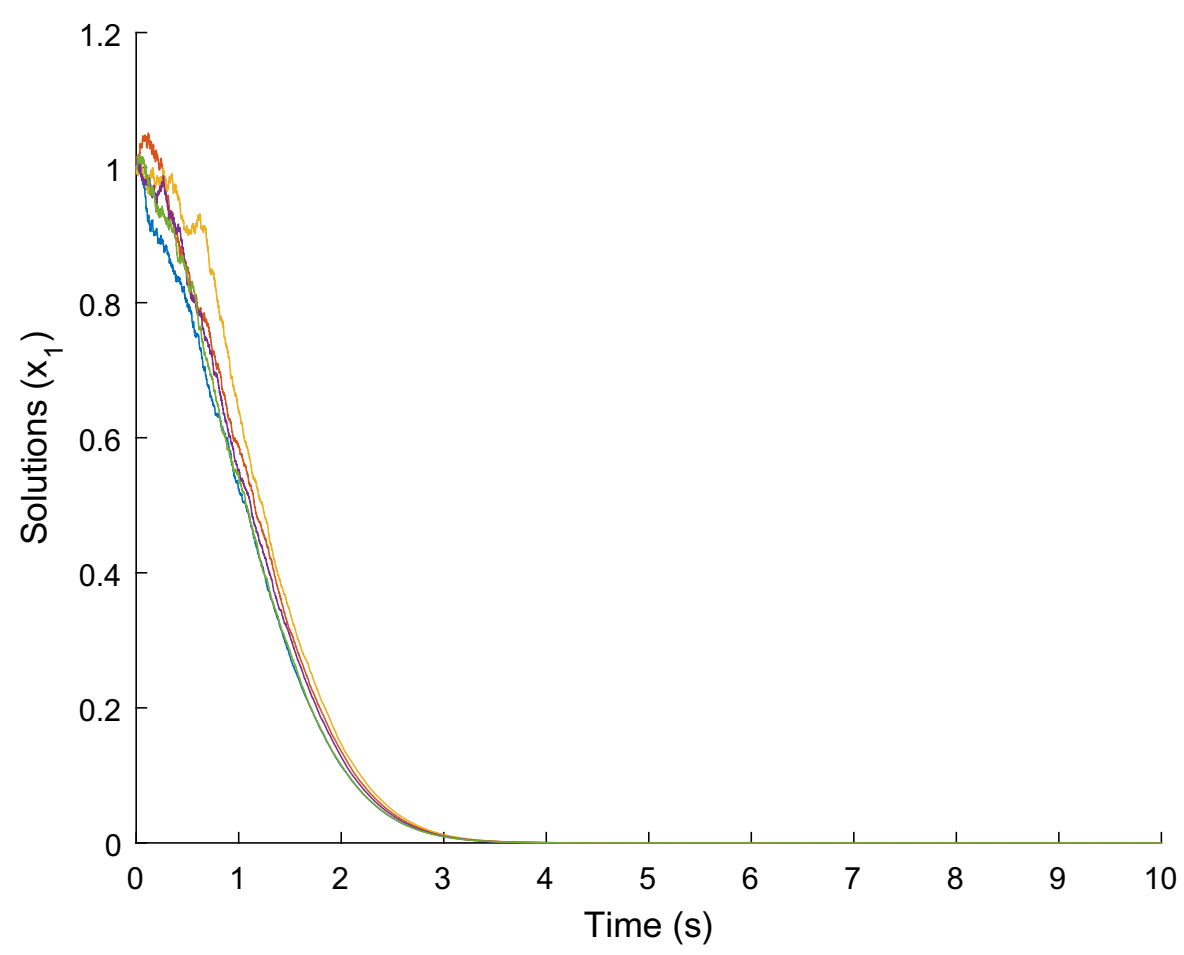

with

$$
\left\{\begin{array}{l}
f_{1}(t, x)=\frac{1}{8} \frac{x_{1}^{2}}{1+\sqrt{x_{1}^{2}+x_{2}^{2}}}+\frac{1}{\sqrt{2}} \sin (2 \pi t) \\
f_{2}(t, x)=\frac{1}{8} \frac{x_{2}^{2}}{1+\sqrt{x_{1}^{2}+x_{2}^{2}}}+\frac{1}{\sqrt{2}} e^{-t} .
\end{array}\right.
$$

System (6.3) can be regarded as a singular perturbed system of the following linear time-varying singular system:

$E(t) d x(t)=A(t) x(t) d t$.

Indeed, $(E, A)$ is transferable into a standard canonical form (SCF) with

$S(t)=\left(\begin{array}{cc}1 & 0 \\ 0 & e^{-t}\end{array}\right), \quad T(t)=\left(\begin{array}{ll}1 & 0 \\ 0 & 1\end{array}\right)$.

Taking into account Proposition 2.1, it follows that $\mathcal{W}=\mathbb{R}_{+} \times \operatorname{im}\left(\begin{array}{l}1 \\ 0\end{array}\right)$.

We select

$$
P(t)=\left(\begin{array}{cc}
\frac{1}{2} & 0 \\
0 & e^{t}
\end{array}\right), \quad Q(t)=\left(\begin{array}{ll}
1 & 0 \\
0 & 1
\end{array}\right) .
$$

One can verify that $P(\cdot)$ and $Q(\cdot)$ solve Eq. (4.3). Thus, we obtain $q_{1}=q_{2}=1$ and $p_{1}=p_{2}=\frac{1}{2}$.

Since, $\operatorname{im} \Pi(t)=\mathcal{W}(t)$, then, for instance, we may choose $\Pi(t)=\left(\begin{array}{ll}1 & 0 \\ 0 & 0\end{array}\right)$.

Hence, we obtain $E \Pi f=\left(\begin{array}{c}f_{1} \\ 0\end{array}\right)$, and $\|f(t, x)\|^{2}=$ $f_{1}^{2}(t, x)+f_{2}^{2}(t, x)$

where $\|$.$\| represents the Euclidean norm.$

Based upon the fact that, $(b+c)^{n} \leq 2^{n-1}\left(b^{n}+\right.$ $\left.c^{n}\right)$, for all $b, c \geq 0, \mathrm{n} \geq 1$, it yields that

$$
\|f(t, x)\|^{2} \leq \frac{1}{32}\left(x_{1}^{2}+x_{2}^{2}\right)+\left(e^{-2 t}+\sin ^{2}(2 \pi t)\right) .
$$

Consequently, we obtain for all $(t, x) \in \mathcal{W}$,

$$
\|f(t, x)\|^{2} \leq \frac{1}{32}\|x\|^{2}+\left(e^{-2 t}+\sin ^{2}(2 \pi t)\right) .
$$

Then, we can choose constants in Theorem 4.4 as follows: $M=1, \gamma=\frac{1}{32}, \phi(t)=\left(e^{-2 t}+\sin ^{2}(2 \pi t)\right)$. It is clear that $q_{1}>p_{2} M^{2} \gamma$ and $\phi(t)$ is a continuous nonnegative bounded function.

Finally, Theorem 4.4 allows us to conclude that the time-varying stochastic perturbed singular system (6.3) 
Fig. 2 The initial response of the system (6.3), with five different Brownian motions, and $x_{0}=[1,0]^{T}$

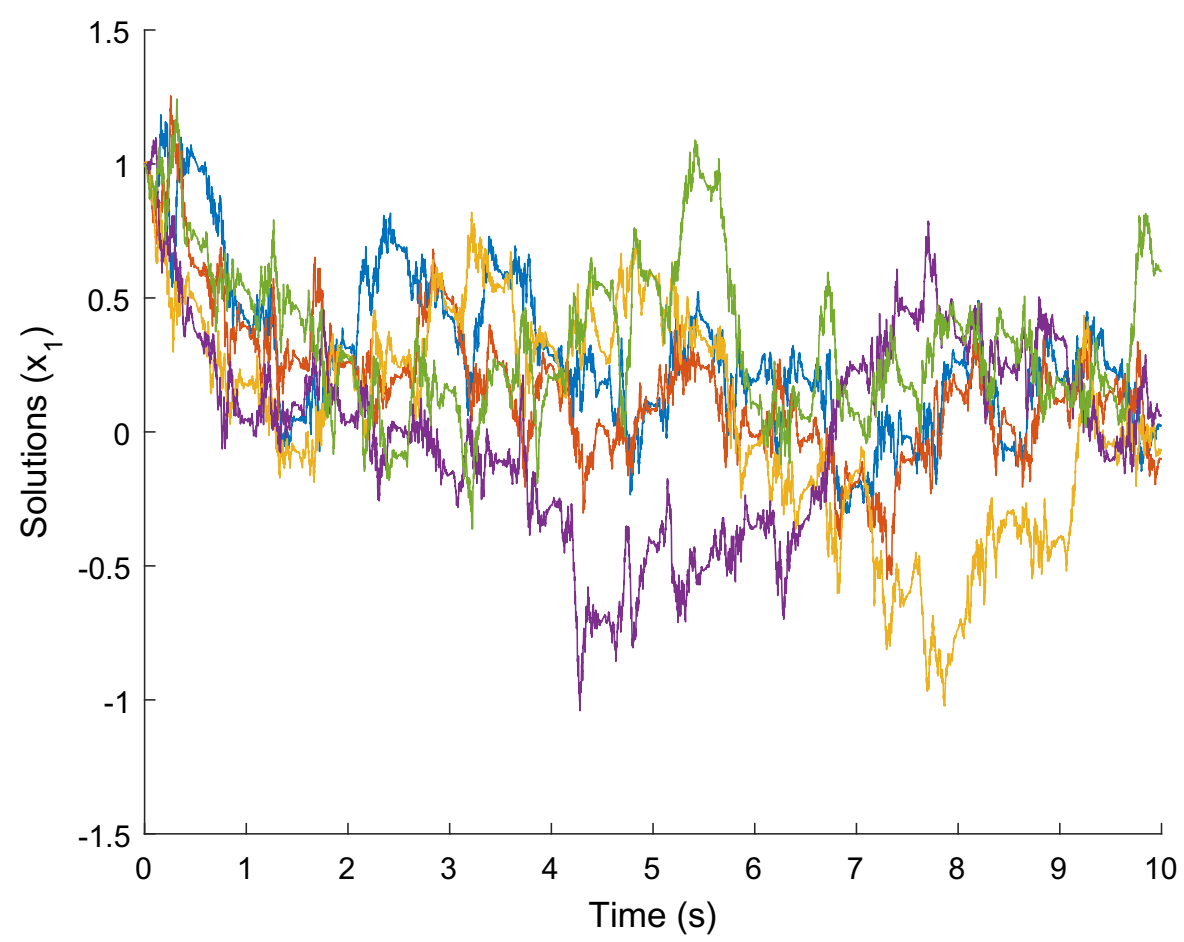

is practically uniformly exponentially stable in mean square, as shown in Figure.2.

\section{Conclusion}

In this paper, we dealt with the stability analysis of some classes of stochastic perturbed singular systems, where their associated unperturbed system is alinear time-varying singular systems transferable into standard canonical forms. Some stability criteria for uniform exponential stability as well as practical uniform exponential stability in mean square of stochastic perturbed singular systems, under the assumption that the initial conditions are consistent, have been established. The main technical tools for deriving stability results are generalized Gronwall inequalities and the direct Lyapunov method. Illustrative examples have been introduced to validate the developed methods. In [11], Caraballo et al. investigated the practical uniform $p$ th moment exponential stability of nonlinear regular stochastic differential equations with respect to a part of the variables. For our next prospect research, we will try to extend our results to the case of $p$ th moment exponential stability of a certain class of time-varying stochastic perturbed singular systems.
Acknowledgements The authors would like to thank the editor and the anonymous reviewer for valuable comments and suggestions, which allowed us to improve the paper.

Data availability statement Data sharing not applicable to this article as no datasets were generated or analyzed during the current study.

\section{Declarations}

Conflict of interest All the authors declare that there are no potential conflicts of interest and approval of the submission.

\section{References}

1. Ben Abdallah, A., Hammami, M.A.: On the output feedback stability for non-linear uncertain control systems. Int. J. Control 6, 547-551 (2001)

2. Ben Abdallah, A., Ellouze, I., Hammami, M.A.: Practical stability of nonlinear time-varying cascade systems. J. Dyn. Control. Syst. 15, 45-62 (2009)

3. Berger, T., Ilchmann, A.: On the standard canonical form of time-varying linear DAEs. Q. Appl. Math. 71, 69-87 (2013)

4. Berger, T.: Bohl exponent for time-varying linear differential algebraic equations. Int. J. Control 10, 1433-1451 (2012)

5. Berger, T., Ilchmann, A.: On stability of time-varying linear differential-algebraic equations. Int. J. Control 86, 1060$1076(2013)$ 
6. Boukass, E.K, Lam, Xuj, : On Stability and stabilizability of singular stochastic systems with delays. J. Optim. Theory Appl. 127, 249-262 (2005)

7. Cong, N.D.: Lyapunov spectrum of nonautonomous linear stochastic differential algebraic equation of index-1. Stoch. Dyn. 4, 1-16 (2012)

8. Cong, N.D., The, N.T.: Stochastic differential algebraic equation of index 1. Vietnam J. Math. 38, 117-131 (2010)

9. Campbell, S.L.: Singular systems of differential equations. Pitman, Marshfield (1980)

10. Caraballo, T., Han, X.: Applied nonautonomous and random dynamical systems. Springer International Publishing, New york (2016)

11. Caraballo, T., Ezzine, F., Hammami, M., Mchiri, L.: Practical stability with respect to a part of variables of stochastic differential equations. Stochastics An International Journal of Probability and Stochastic Processes 1-18,(2021)

12. Caraballo, T., Ezzine, F., Hammami, M.A.: On the exponential stability of stochastic perturbed singular systems in mean square. Appl Math Optim (2021). https://doi.org/10. 1007/s00245-020-09734-8

13. Dai, L.: Singular Control systems. Springer, New york (1989)

14. Debeljkovic, D.. Lj.., Jovanović, B.., Drakulić, V..: Singular system theory in chemical engineering theory: Stability in the sense of Lyapunov: A survey. Hemijska Industrija 6, 260-272 (2001)

15. D. Lj. Debeljkovic, D. H. Ownes, Consistency and Liapunov Stability of Linear Descriptor systems: A Geometric Analysis, IMA Journal of Mathematical Control and Information, 2, 139-151 (1985)

16. Jiang, X., Tian, S., Zhang, T., Zhang, W.: Stability and stabilization of nonlinear discrete-time stochastic systems. Int. J. Robust Nonlinear Control 29, 6419-6437 (2019)

17. T. Jiao, Ju H. Park, G, Zong, Stability criteria of stochastic nonlinear systems with asyn-chronous impulses and switchings, Nonlinear Dynamics, 97, 135-149 (2019)

18. Khasminskii, R.: Stochastic stability of differential equations, Stochastic Modelling and Applied Probability 66, 2nd edition, (2012)

19. Kunkel, P., Mehrmann, V.: Differential-algebraic equations analysis and numerical solution. EMS Publishing House, Zurichd (2006)
20. Mao, X.: Stochastic differential equations and applications. Ellis Horwood, Chichester (1997)

21. Mao, X.: Stochastic stabilization and destabilization. Syst. Control Lett. 23, 279-290 (1994)

22. Pham, Q.C.: A variation of Gronwall's lemma arXiv 13,(2007)

23. Ritt, J.F.: Systems of algebraic differential equations. Ann. Math. 36, 293-302 (1935)

24. Sathananthan, S., Knap, M.J., Strong, A., Keel, L.H.: Robust stability and stabilization of a class of nonlinear discrete time stochastic systems: An LMI approach. Appl. Math. Comput. 219, 1988-1997 (2012)

25. Scheutzow, M.: Stabilization and destabilization by noise in the plane. Stoch. Anal. Appl. 1, 97-113 (1993)

26. Winkler, R.: Stochastic differential algebraic equations of index 1 and applications in circuit simulation. J. Comput. Appl. Math. 163, 435-463 (2004)

27. Xu, Y., Ying Wang, Xi., Qing Zhang, H., Xu, W.: Stochastic stability for nonlinear systems driven by Lévy noise, Nonlinear Dynamics, 68, 7-15 (2012)

28. Yan, Z., Zhang, W.: Finite-Time Stability and Stabilization of Itô-Type Stochastic Singular Systems. Abstract And Applied analysis 1-10,(2014)

29. Zhang, Q., Xing, S.: Stability analysis and optimal control of stochastic singular systems. Optim. Lett. 8, 1905-1920 (2014)

30. Zhang, W., Zhao, Y., Sheng, L.: Some remarks on stability of stochastic singular systems with state-dependent noise. Automatica 51, 273-277 (2015)

Publisher's Note Springer Nature remains neutral with regard to jurisdictional claims in published maps and institutional affiliations. 